\title{
NOUVELLe
}

\section{Implication de miR-199a-5p dans la fibrose pulmonaire idiopathique}

Imène Sarah Henaoui ${ }^{1}$, Christelle Cauffiez ${ }^{2}$, Sébastien Aubert ${ }^{3}$, Matthieu Buscot ${ }^{1,4}, \varepsilon d m o n e$ Dewaeles ${ }^{2}$, Marie-Christine Copin ${ }^{3}$, Charles-Hugo Marquette ${ }^{4}$, Pascal Barbry ${ }^{1}$, Michael Perrais ${ }^{5}$, Nicolas Pottier ${ }^{2}$, Bernard Mari ${ }^{1}$
IUMR-7275 CNRS, université de Nice Sophia-Antipolis, institut de pharmacologie moléculaire et cellulaire, 660 , route des Lucioles Sophia-Antipolis, 06560 Valbonne, Nice, France ;

${ }^{2} \varepsilon$ A4483, faculté de médecine de Lille, pôle recherche, 1, place de Verdun, 59045 Lille, France ; ${ }^{3}$ Pôle de pathologie, CHRU de Lille, Lille, France ;

${ }^{4}$ Service de pneumologie, CHRU de Nice, Nice, France;

${ }^{5}$ Inserm U837, centre Jean-Pierre Aubert, Lille, France. nico_pottier@yahoo.fr henaoui@ipmc.cnrs.fr
La fibrose pulmonaire idiopathique Les pathologies fibroprolifératives représentent un problème majeur de santé publique et leur prévalence est en constante augmentation dans les pays industrialisés [1]. La fibrose pulmonaire idiopathique (FPI) est une forme fréquente et sévère de pneumopathie interstitielle idiopathique diffuse. Elle évolue souvent vers une insuffisance respiratoire progressive, et la médiane de survie est inférieure à cinq ans une fois le diagnostic établi [2]. La fibrose pulmonaire idiopathique est une pathologie chronique rare dont les causes demeurent mal connues, et qui ne bénéficie à ce jour d'aucun traitement efficace. Elle se caractérise histologiquement par un aspect de pneumopathie interstitielle commune, correspondant à une fibrose hétérogène et mutilante de l'interstitium pulmonaire avec des foyers de prolifération fibroblastique, des lésions en rayons de miel et de rares lésions inflammatoires (Figure 1). Le diagnostic repose sur l'analyse pluridisciplinaire des symptômes cliniques, la tomodensitométrie thoracique, l'absence de signes biologiques en faveur d'une maladie systémique et la biopsie pulmonaire vidéochirurgicale lorsqu'elle est indiquée. La fibrose correspond au dépôt excessif et persistant de tissu conjonctif cicatriciel en réponse à une lésion tissulaire chronique qui aboutit à la perte de fonction de l'organe atteint. Sur le plan physiopathologique, on considère actuellement qu'une agression répétée de l'épithélium alvéolaire serait responsable de la sécrétion par les pneumocytes de facteurs de croissance, tels que le TGF $\beta$ (transforming growth factor $\beta$ ), qui favoriseraient le recrutement, la prolifération et l'activation de fibroblastes pulmonaires ainsi que le dépôt excessif de matrice extracellulaire. Lors du processus de fibrose, les fibroblastes, organisés en foyers, acquièrent un phénotype de myofibroblastes. D’autres mécanismes pourraient également participer au processus de fibrose comme la transition épithélio-mésenchymateuse des cellules épithéliales, endothéliales ou mésothéliales, ainsi que le recrutement pulmonaire de fibrocytes circulants d'origine médullaire [2].

Les microARN :

une nouvelle classe

de régulateurs de l'expression génique Les microARN (miARN) sont des petits ARN non codants d'environ 22 bases dont le rôle clé dans la régulation de nombreux processus cellulaires - survie, apoptose, prolifération, ou différenciation - a été récemment documenté [3]. Leurs mécanismes d'action

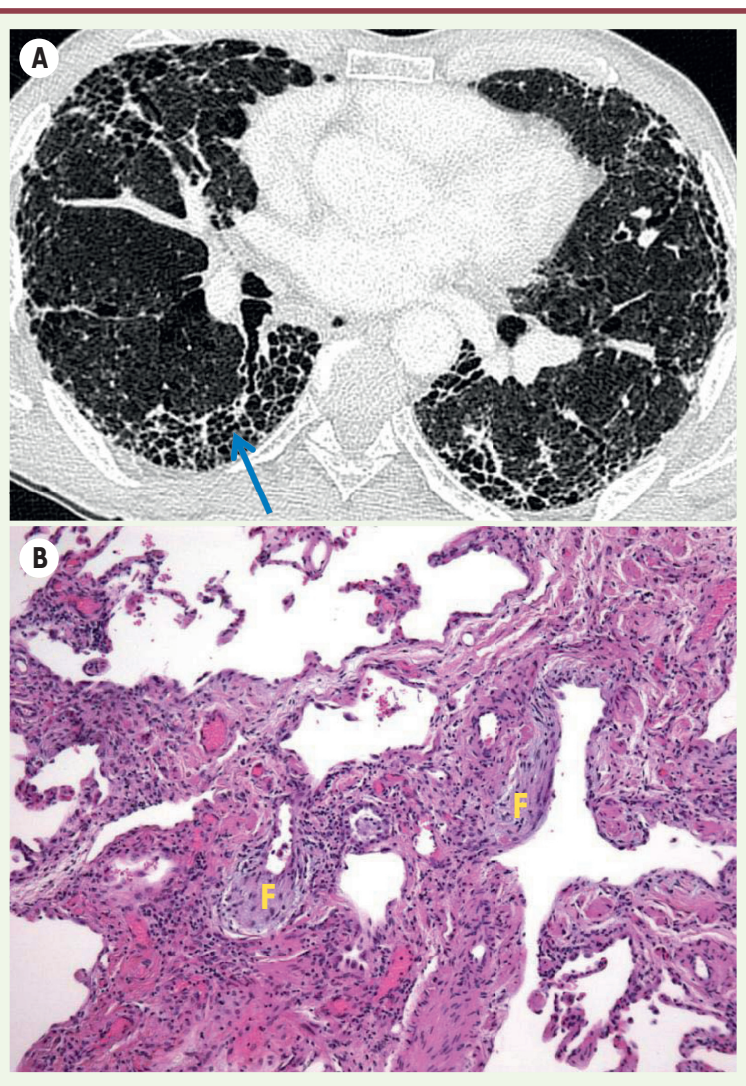

Figure 1. Aspects radiographiques et histologiques de la fibrose pulmonaire idiopathique. A. Tomodensitométrie thoracique montrant un aspect en rayon de miel typique de la fibrose pulmonaire idiopathique (flèche). B. Aspect histologique de fibrose pulmonaire idiopathique sur coupe de poumon colorée à l'hématoxyline/ éosine. Observation à faible grossissement montrant notamment la présence de foyers fibroblastiques (F).

impliquent généralement la formation d'un complexe entre plusieurs bases situées dans la région 5' du miARN (séquence de reconnaissance ou seed) et l'ARNm cible (principalement dans sa partie 3' non codante [4]. 


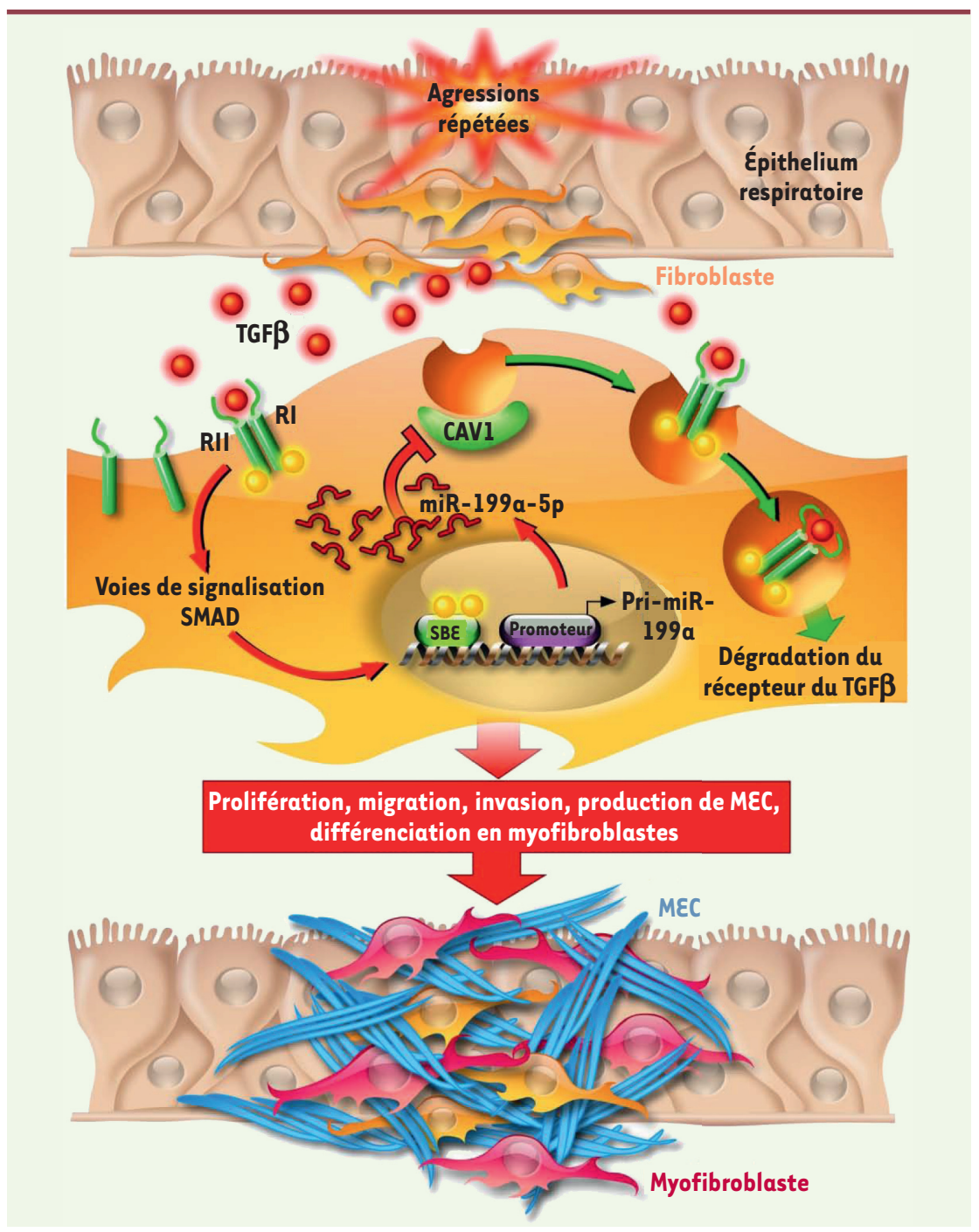

Figure 2. Modèle proposé pour la fonction de miR-199a-5p dans la fibrose pulmonaire. Les microlésions itératives de l'épithélium pulmonaire induisent un relargage de TGF $\beta$ qui se lie aux récepteurs TGF $\beta R$ ( $R I$ et RII) au niveau des fibroblastes pulmonaires et induit l'expression de miR-199a-5p. Le complexe résultant sera internalisé selon deux voies distinctes d'endocytose. La voie dépendante de la clathrine (flèches rouges) est responsable d'une augmentation de la transduction du signal induite par le TGF $\beta$, d'une activation des fibroblastes pulmonaires et de leur différenciation en myofibroblastes. La voie des radeaux lipidiques et des cavéoles (flèches vertes) (voie indépendante de la clathrine) induit quant à elle une dégradation du complexe TGF $\beta / T G F \beta R$, inhibant de ce fait le processus de fibrose. La production de miR-199a-5p induit une régulation négative de la production de cavéoline-l qui active alors la voie du TGF $\beta$ et génère des conséquences pathologiques. CAVI : cavéoline-1; TGF $\beta$ : transforming growth factor- $\beta$; TGF $\beta$ R : transforming growth factor- $\beta$ receptor ; SBE : SMAD binding element ; MEC : matrice extracellulaire (adapté de [7]).

La reconnaissance étant contrôlée par une courte séquence d'environ sept bases, chaque miARN a de facto la capacité de réguler un grand nombre de transcrits distincts. La maturation des miARN s'effectue tout d'abord dans le noyau, via les enzymes Drosha et Dicer. Les miARN sont ensuite
miR-199a-5p : un «fibromiR » surexprimé dans la fibrose pulmonaire Plusieurs miARN ont été impliqués dans les processus de fibrose tissulaire, mais ni leur(s) rôle(s) précis, ni leur(s) mécanisme(s) d'action n'ont été clairement définis [5]. Dans un travail publié récemment dans Plos Genetics [6], nous avons déterminé le profil d'expression pulmonaire des miARN dans un modèle expérimental murin de fibrose pulmonaire induite par la bléomycine, couramment utilisé dans l'étude de la fibrose pulmonaire idiopathique. De manière intéressante, un microARN, miR-199a-5p, est surexprimé lors du processus de fibrose ; miR-199a-5p apparaissait comme le meilleur candidat car, d'un point de vue statistique, les variations de son expression étaient plus importantes dans les souches sensibles que dans les souches résistantes à la bléomycine. Sa surexpression a ensuite été confirmée dans des échantillons pulmonaires issus de patients atteints de fibrose pulmonaire idiopathique, mais aussi par hybridation in situ au sein des foyers fibroblastiques. Ces résultats évoquaient une implication possible de miR-199a-5p dans la physiopathologie de cette maladie, en particulier dans l'activation des fibroblastes pulmonaires et leur différenciation en myofibroblastes. En effet, la surexpression de miR-199a-5p dans les fibroblastes pulmonaires induit une augmentation des capacités prolifératives, migratrices et invasives de ces cellules, ainsi que leur différenciation en myofibroblastes.

\section{miR-199 $a-5 p$ régule négativement} l'expression de CAV1, essentielle à la réponse des fibroblastes pulmonaires au TGF $\beta$

Les gènes cibles de miR-199a-5p ont ensuite été identifiés par l'étude du transcriptome des fibroblastes pulmonaires dans lesquels miR-199a-5p avait été surexprimé. Une diminution significative de l'expression du gène codant pour la cavéoline-1 (CAVI), un puissant inhibiteur du processus de fibrose pulmonaire, a été identifiée. 
CAVl est une protéine membranaire de 22 kDa essentielle à la formation des petites invaginations de la membrane plasmique, les caveolae. Les cavéoles représentent une catégorie de radeaux lipidiques particulièrement abondante dans les cellules différenciées comme les adipocytes, les cellules endothéliales, les pneumocytes de type I, les fibroblastes et les cellules musculaires lisses et striées. Dans le contexte d'un processus de fibrose pulmonaire, de nombreuses études ont montré l'implication des caveolae dans l'internalisation et la dégradation du complexe formé par le TGF $\beta$ et ses récepteurs. II est en effet bien établi que l'activation des fibroblastes pulmonaires par le TGF $\beta$ induit une diminution de l'expression de CAVl et une perte des signaux inhibiteurs exercés par les cavéoles sur la voie de signalisation du TGF $\beta$ [7].

Or, nos résultats montrent que l'exposition des fibroblastes pulmonaires au TGF $\beta$ induit la surexpression de miR-199a-5p ainsi qu'une diminution concomitante de l'expression de CAVI. Deux approches indépendantes basées sur l'utilisation d'oligonucléotides soit dirigés contre miR199a-5p et permettant son inhibition, soit conçus pour bloquer spécifiquement la fixation du miARN sur sa cible CAVI, ont permis de démontrer le rôle de miR$199 a-5 p$ dans la voie de signalisation du TGF $\beta$. En effet, La transfection de ces deux types d'oligonucléotides dans les fibroblastes pulmonaires inhibe l'activation et la différenciation en myofibroblastes des fibroblastes traités par le TGF $\beta$. L'ensemble de ces résultats suggèrent que miR-199a-5p participe à la régulation de CAVl et représente un élément essentiel de la réponse des fibroblastes pulmonaires au TGF $\beta$ (Figure 2) [6].

\section{Perspectives thérapeutiques}

Notre étude révèle pour la première fois le rôle clé joué par le fibromiR miR199a-5p dans le processus de fibrose pulmonaire. En particulier, ce miARN apparaît comme un régulateur essentiel de la signalisation du TGF $\beta$, notamment par ses effets sur le gène codant pour la cavéoline-1. Ces résultats pourraient susciter de nouvelles stratégies thérapeutiques dans le cadre des pathologies fibroprolifératives basées sur la modulation de l'expression des miARN à l'aide d'oligonucléotides de synthèse. Une telle approche a déjà été utilisée avec des résultats significatifs dans le traitement de l'hépatite C [8]. Ainsi, le développement de molécules dirigées contre les fibromiR pourrait permettre à terme de stopper la progression de la fibrose, voire de la faire régresser. $\diamond$ miR-199a-5p in idiopathic pulmonary fibrosis

\section{LIENS D'INTÉRÊT}

Les auteurs déclarent n'avoir aucun lien d'intérêt concernant les données publiées dans cet article.

\section{RÉFÉRENCES}

1. Wynn TA. Common and unique mechanisms regulate fibrosis in various fibroproliferative diseases. J Clin Invest 2007 ; 117 : 524-9.

2. Wilson MS, Wynn TA. Pulmonary fibrosis: pathogenesis, etiology and regulation. Mucosal Immunol 2009 ; 2 : $103-21$.

3. Hartmann C, Corre-Menguy F, Boualem A, et al. Les microARN. Med Sci (Paris) $2004 ; 20: 894-8$.

4. Brennecke J, Stark A, Russell RB, et al. Principles of microRNA-target recognition. PLoS Biol 2005 ; 3 : e85

5. Pandit KV, Milosevic J, Kaminski N. MicroRNAs in idiopathic pulmonary fibrosis. Transl Res 2011 ; 157 : 191-9.

6. Lino Cardenas CL, Henaoui IS, Courcot $\varepsilon$, et al. miR$199 a-5 p$ is upregulated during fibrogenic response to tissue injury and mediates TGFbeta-induced lung fibroblast activation. PLoS Genet 2013 ; 9 : e1003291.

7. Wang XM, Zhang Y, Kim HP, et al. Caveolin-1: a critical regulator of lung fibrosis in idiopathic pulmonary fibrosis.J Exp Med 2006 ; 203 : 2895-906.

8. Lanford RE, Hildebrandt-Eriksen ES, Petri A, et al. Therapeutic silencing of microRNA-122 in primates with chronic hepatitis C virus infection. Science 2010 ; 327 : 198-201.

\section{NOUVELLE}

\section{La métabolomique au service de la médecine}

\section{L’exemple du carcinome rénal}

Valérian Dormoy ${ }^{1,2}$, Thierry Massfelder ${ }^{1}$

Un nouveau membre de la famille

\section{«-omique 》}

La métabolomique, qui représente une discipline nouvelle au sein de la grande famille «-omique», comporte de nouveaux outils à fort potentiel et suscite un vif intérêt dans la communauté scientifique, alors que les contraintes techniques semblaient infranchissables il y a seulement quelques années $[1,2]$.
Que se cache-t-il derrière ce terme de métabolomique, élevé récemment au rang de science (Figure l) ? Un métabolite est une substance de petite taille (en général moins d'un kilodalton) formée au cours du métabolisme, qu'il s'agisse du produit final ou d'un intermédiaire. La principale limitation à l'identification des métabolites se situe dans l'usage des techniques de détection. II est clas-
${ }^{1}$ Inserm U1113, équipe 3

«signalisation et communication cellulaires dans les cancers du rein et de la prostate », université de Strasbourg, faculté de médecine, 11, rue Humann, 67085 Strasbourg, France ; ${ }^{2}$ Département de biologie développementale et cellulaire, université de Californie, Irvine, 4150 McGaugh Hall, 92697 Irvine, CA, États-Unis. valerian.dormoy@unistra.fr massfeld@unistra.fr

sique de classer ces métabolites en deux catégories en fonction de leur origine: endogènes lorsqu'ils sont produits par l'organisme, exogènes lorsqu'ils proviennent de l'environnement extérieur. La métabolomique, en tant que technique, apparaît au tout début du 